\title{
A GENERALIZED INVERSION FORMULA FOR THE CONTINUOUS JACOBI TRANSFORM
}

\author{
AHMED I. ZAYED \\ Department of Mathematics \\ California Polytechnic State University \\ San Luis Obispo, CA $934 n 7$ \\ U.S.A.
}

(Received August 19, 1986 and in revised form September 10, 1986)

ABSTRACT. In this paper we extend the definition of the continuous Jacobi transform to a class of generalized functions and obtain a generalized inversion formula for it. As a by-product of our technique we obtain a necessary and sufficient condition for an analytic function $F(\lambda)$ in $\operatorname{Re} \lambda>0$ to be the continuous Jacobi transform of a generalized function.

KEY WORDS AND PHRASES. Continuous Jacobi transform, Generalized functions, Inverse Jacobi transform.

1980 AMS SUBJECT CLASSIFICATION CODE. 44A15, 33A65 and 33A3.

1. Introduction:

The main principle in sampling theory is to reconstruct a function $f(t)$ defined on some open interval I from the knowledge of its values $f\left(t_{n}\right)$ at a discrete sequence of points $\left\{t_{n}\right\}_{n=0}^{\infty} \subset I$. In Kramer's sampling theorem [9], this can be expressed in the form

$$
f(t)=\sum_{n=0}^{\infty} f\left(t_{n}\right) s_{n}(t) ; t_{\varepsilon} I
$$

where $\left\{S_{n}(t)\right\}_{n=0}^{\infty}$ is a sequence of determined functions.

Suppose that $\left\{\phi_{n}(t)_{n=0}^{\infty}\right.$ is a complete system of orthonormal functions defined on I with respect to some measure $d_{p}(t)$. Let us call these functions p-functions. In the case when $f(t)$ can be expanded in terms of the p-functions,i.e.,

$$
f(t) \sum_{n=0}^{\infty} a(n) \phi_{n}(t)
$$

where

$$
a(n)=\int_{I} f(t) \phi_{n}(t) d \rho(t)
$$

determining $\left\{S_{n}(t)\right\}_{0}^{\infty}$ requires generalizing the $p$-functions to the case where 
the discrete parameter $n$ is replaced by a continuous real parameter $\lambda$ in some open interval $\mathrm{J}$.

This has motivated the idea of generalizing various classical orthonormal sequences of functions to the continuous parameter case which in turn has led to the investigation of expanding functions in terms of the generalized p-functions $\left\{\phi_{\lambda}(t)\right\}_{\lambda \varepsilon J}$ If we call (1.3) the discrete p-transform of $f(t)$ and regard (1.2) as its inversion formula, then analogously we can define the continuous p-transform of $f(t)$ as

$$
a(\lambda)=\int_{I}^{\infty} f(t) \phi_{\lambda}(t) d \rho(t)
$$

But since the $\phi_{\lambda}(t)$ 's are no longer orthogonal, finding an inversion formula for the continuous p-transform (1.4) is usually more difficult.

An explicit iepreselitation of $\left\{S_{n}(t)\right\}_{0}$ has been given in [2 ], [7] and [8] for the cases where the $p$-functions are the Bessel's functions, Legendre, associated Legendre, Gegenbauer, Chebyshev, Laguerre polynomials. However, only in a very few cases an inversion formula for the continuous transform is known. Inversion formulas for the continuous Legendre and the continuous Jacobi transforms were derived by Butzer, Stens, Wehrens [1 ] and Deeba, Koh [5] respectively.

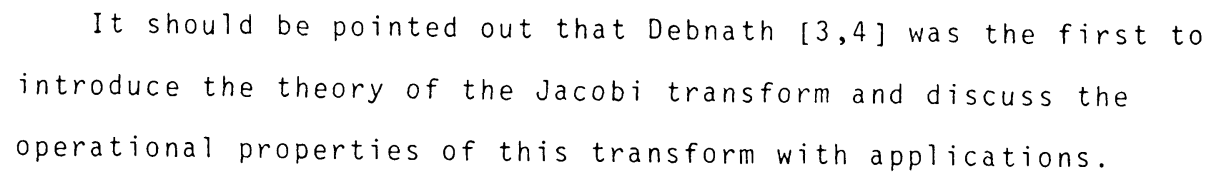

The main purpose of this paper is to derive a more general inversion formula for the continuous Jacobi transform which will include the results of [1 ] and [5] as a special case. As a by-product of our approach we obtain a more general definition of the continuous Jacobi transform which enables us to give a necessary and sufficient condition for an analytic function $F(\lambda)$ in $\operatorname{Re} \lambda>0$, to be the continuous Jacobi transform of a generalized function $f(t)$.

2. Prel iminaries:

In this section we recall some of the basic background material necessary for our investigation.

For any real numbers $a, b$ and $c$ with $c \neq 0,-1,-2, \ldots$, the hypergeometric function $F(a, b ; c ; z)={ }_{2} F_{1}(a, b ; c ; z)$ is given by 


$$
F(a, b ; c ; z)=\sum_{k=0}^{\infty} \frac{(a)_{k}(b)_{k}}{(c)_{k} k !} z^{k},|z|<1
$$

where the series converges at $z=-1$ and $z=1$ provided that $c-a-b+1>0$ and $c-a-b>0$ respectively.

The Jacobi function $\mathrm{p}_{\lambda}^{(\alpha, \beta)}(x)$ of the first kind is defined by

$$
P_{\lambda}^{(\alpha, \beta)}(x)=\frac{\Gamma(\lambda+\alpha+1)}{\Gamma(\alpha+1) \Gamma(\lambda+1)} F\left(-\lambda, \lambda+\alpha+\beta+1 ; \alpha+1 ; \frac{1-x}{2}\right), \quad x \in(-1,1]
$$

where $a, s>-1, \lambda \in \mathbb{R}$ and $\lambda+\alpha+1 \neq 0,-1,-2, \ldots$. Since

$$
p_{-\lambda}^{(\alpha, \beta)}(x)=\frac{\Gamma(\alpha-\lambda+1) \Gamma(\lambda-\alpha-\beta)}{\Gamma(1-\lambda) \Gamma(\lambda-\beta)} p_{\lambda-\alpha-\beta-1}^{(\alpha, \beta)}(x)
$$

we may restrict ourselves to the case $\lambda \geq-\left(\frac{\alpha+\beta+1}{2}\right)$. The function $p_{\lambda}^{(\alpha, \beta)}(x)$ satisfies the differential equation

$$
\left(1-x^{2}\right) y^{\prime \prime}+(\beta-\alpha-(\alpha+\beta+2) x) y^{\prime}+\lambda(\lambda+\alpha+\beta+1) y=0 .
$$

Let

$$
p_{\lambda}^{(\alpha, \beta)}(x)=(1-x)^{\alpha}(1+x)_{\lambda}^{\beta} p_{\lambda}^{(\alpha, \beta)}(x)
$$

and

$$
L=\left(1-x^{2}\right) \frac{d^{2}}{d x^{2}}+[(\alpha-\beta)+(\alpha+\beta-2) x] \frac{d}{d x}+(\alpha+\beta)
$$

Then with some easy computations, one can show that $p_{\lambda}^{(\alpha, \beta)}(x)$ are the eigenfunctions of $L$ with eigenvalues $-\lambda(\lambda+\alpha+\beta+1)$, i.e.

$$
L p_{\lambda}^{(\alpha, \beta)}(x)=-\lambda(\lambda+\alpha+\beta+1) p_{\lambda}^{(\alpha, \beta)}(x)
$$

One can also verify that the conjugate differential operator $L^{\star}$ of $L$ is given by

$$
L^{*}=\left(1-x^{2}\right) \frac{d^{2}}{d x^{2}}-[(\alpha-\beta)+(\alpha+\beta+2) x] \frac{d}{d x} .
$$


It can be shown that for $\lambda, \nu \geq-\left(\frac{\alpha+\beta+1}{2}\right), \lambda \neq \nu, \lambda \neq-(\nu+\alpha+\beta+1)$ and $-1<\alpha, \quad \beta$

$$
\begin{aligned}
& \frac{1}{2^{\alpha+\beta+1}} \int_{-1}^{1}(1-x)^{\alpha}(1+x)_{\lambda}^{\beta_{p}(\alpha, \beta)}(x) P_{\nu}^{(\beta, \alpha)}(-x) d x \\
= & \frac{\Gamma(\lambda+\alpha+1) \Gamma(\nu+\beta+1)}{\pi(\lambda-\nu)(\lambda+\nu+\alpha+\beta+1)}\left\{\frac{\sin \pi \lambda}{\Gamma(\nu+1) \Gamma(\lambda+\alpha+\beta+1)}-\frac{\sin \pi \nu}{\Gamma(\lambda+1) \Gamma(\nu+\alpha+\beta+1)}\right\} .
\end{aligned}
$$

If we denote the Jacobi polynomial of degree $n$ by $p_{n}^{(\alpha, \beta)}(x)$, then

$\therefore r=r e$

$$
\frac{1}{2^{\alpha+\beta+1}} \int_{-1}^{1}(1-x)^{\alpha}(1+x)^{\beta_{p}}{ }_{n}(\alpha, \beta)(x) P_{m}^{(\alpha, \beta)}(x) d x=\delta_{n m} h_{n}
$$

$$
h_{n}=\frac{\Gamma(n+\alpha+1) \Gamma(n+\beta+1)}{n !(2 n+\alpha+\beta+1) \Gamma(n+\alpha+\beta+1)} .
$$

Since $P_{n}^{(\alpha, \beta)}(-x)=(-1)^{n_{p}(\beta, \alpha)}(x)$ it follows that

$$
\begin{aligned}
\hat{p}_{\lambda}^{(\alpha, \beta)}(n) & =\frac{1}{2^{\alpha+\beta+1}} \int_{-1}^{1}(1-x)^{\alpha}(1+x)_{\lambda}^{\beta} p_{\lambda}^{(\alpha, \beta)}(x) P_{n}^{(\alpha, \beta)}(x) d x \\
& =\frac{(-1)^{n} \Gamma(\lambda+\alpha+1) \Gamma(n+\beta+1) \sin \pi \lambda}{\pi(\lambda-n)(\lambda+n+\alpha+\beta+1) n ! \Gamma(\lambda+\alpha+\beta+1)}, \quad \lambda \neq n
\end{aligned}
$$

and hence

$$
p_{\lambda}^{(\alpha, \beta)}(x) \sim \sum_{n=0}^{\infty} \frac{1}{h_{n}} \hat{p}_{\lambda}^{(\alpha, \beta)}(n) P_{n}^{(\alpha, \beta)}(x), \quad x \in(-1,1] .
$$

The following estimates will also be needed:

$$
\begin{aligned}
& \text { i) for }-1<\beta \leq 0, \sup _{x \in[-1,1]}\left|(1+x) c_{\lambda} p_{\lambda}(\alpha, \beta)(x)\right|<\infty \text {, for any } c>0 \\
& \text { ii) for } \beta \geq 0, \sup _{x \in[-1,1]}\left|(1+x)^{\beta+c_{p}(\alpha, \beta)}(x)\right|<\infty \text {, for any } c>0 \\
& \text { iii) } p_{\lambda}^{(\alpha, \beta)}(x)=0\left(\frac{1}{\sqrt{\lambda}}\right) \text { as } \lambda \rightarrow \infty \text { uniformly in } x \in[a, b] c(-1,1)
\end{aligned}
$$

iv) as a function in $\lambda$ the supremum $k(\lambda)$ in (2.13) and (2.14) is at most of polynomial growth as $\lambda \rightarrow \infty$. In fact,

$$
k(\lambda)=O\left(\lambda^{q}\right) \text { as } \lambda \rightarrow \infty \text { where } q=\max (\alpha, \beta) \text {. }
$$


If $X$ denotes a locally convex space, then its dual will be denoted by $x^{\star}$ and the action of $f \in X^{\star}$ on any member $\phi \varepsilon X$ :ill be denoted by $\langle f, \phi\rangle$.

Let $I=(a, b), \quad-\infty \leq a<b \leq \infty$ anci $C^{\infty}(I)$ be the class of all infinitely differentiable functions on $I$. The space of all infinitely differentiable functions on I provided with its standard topology as described in [10] will be denoted by $E(I)$ while $D(I)$ will denote the space of all functions $\phi(x) \in C^{\infty}(I)$ whose support is compact and lies entirely inside $I$. The topology of $D(I)$ is its standard topology as described in [ 8 ].

Let $w(x)=(1-x)^{\alpha}(1+x)^{\beta}$ and $f(x) \in L^{p}\{(-1,1), w(x)\}, p \geq 1$. Then the discrete Jacobi transform $\hat{f}^{(\alpha, \beta)}(n)$ of $f(x)$ is defined by

$$
\hat{f}^{(\alpha, \beta)}(n)=\frac{1}{2^{\alpha+\beta+1}} \int_{-1}^{1}(1-x)^{\alpha}(1+x)^{\beta} p_{n}(\alpha, \beta)(x) f(x) d x
$$

and the series expansion of $f(x)$ in terms of the Jacobi polynomials is given by

$$
f(x) \sim \sum_{n=0}^{\infty} \frac{1}{h_{n}} \hat{f}^{(\alpha, \beta)}(n) P_{n}^{(\alpha, \beta)}(x), \quad x \in(-1,1) .
$$

Analogously, if $\alpha>-1, \quad-1<\beta<1$ and $f(x) \in L^{2}\{(-1,1), w(x)\}$ the continuous Jacobi transform $\hat{f}^{(\alpha, \beta)}(\lambda)$ of $f(x)$ will be defined by

$$
\hat{f}^{(\alpha, \beta)}(\lambda)=\frac{1}{2^{\alpha+\beta+1}} \int_{-1}^{1}(1-x)^{\alpha}(1+x)^{\beta} p_{\lambda}^{(\alpha, \beta)}(x) f(x) d x ; \lambda>-\frac{1}{2} .
$$

It can be shown that for $-1<\alpha,-1<\beta<1$ and $\alpha+\beta=0$, if $f(x) \varepsilon L^{2}\{(-1,1), w(x)\}$ and $\sqrt{\lambda} \hat{f}(\alpha, \beta)\left(\lambda-\frac{1}{2}\right) \in L^{1}(0, \infty)$, then

$$
f(x)=4 \int_{0}^{\infty} \hat{f}(\alpha, \beta)\left(\lambda-\frac{1}{2}\right) P_{\lambda-\frac{1}{2}}^{(\beta, \alpha)}(-x) H_{0}(\lambda) \lambda \sin \pi \lambda d \lambda
$$

almost everywhere in $(-1,1)$ where

$$
H_{0}(\lambda)=\frac{\Gamma^{2}\left(\lambda+\frac{1}{2}\right)}{\Gamma\left(\lambda+\alpha+\frac{1}{2}\right) \Gamma\left(\lambda+\beta+\frac{1}{2}\right)} .
$$


In addition, if $f(x)$ is continuous on $(-1,1)$, then (2.20) will hold everywhere on $(-1,1)$.

Here we should point out that in [5], formulas (2.19) and $(2.20)$ were proved for $-\frac{1}{2}<\alpha, \beta<\frac{1}{2}$, however they can be easily extended to the case where $-1<\alpha, \beta<1$ since $(1+x)^{\beta}(1-x)^{\alpha}\left|P_{\lambda}^{(\alpha, \beta)}(x)\right|^{2}$ is integrable over $(-1,1)$. Similar argument can be applied to (2.8).

3. Jacobi transforin of a class of generalized functions:

Throughout the rest of this paper I will denote the open interval $(-1,1)$. Fix $\alpha$ and $\beta$ such that $\alpha, \beta>-1$. We define the space $H_{\alpha, \beta}$ as the space of all infinitely differentiable functions $\phi(x)$ defined on I such that

$$
\gamma_{k, c}(\phi)=\sup _{x \in I}\left|(1-x)^{-\alpha}(1+x)^{c} L_{\phi}^{k}(x)\right|<\infty
$$

for any nonnegative integer $k$ and any $c$ such that $\max (-B, 0)<c<1$.

Clearly, $H_{\alpha, \beta}$ is a linear space under the pointwise addition of functions and multiplication by complex numbers. The collection $\Gamma=\left\{\gamma_{k, c}\right\}_{k=0, \max }^{\infty}(-\beta, 0)<c<1$ is a separating family of seminorms since each $\gamma_{k, c}(k=1,2, \ldots)$ is a seminorm and $\gamma_{0, c}$ is a norm. The topology of $H_{\alpha, \beta}$ is the topology induced by the family $\Gamma$. In fact, it is not hard to show that the same topology can be generated by a countable subset of $\Gamma$. Provided with this topology, $H_{\alpha, B}$ becomes a locally convex, Hausdorff topological vector space. By using an argument similar to the one used in lemma 3.2-1 in [ 8], one can show that $H_{\alpha, \beta}$ is, in fact,a Fréchet space and in addition to that $D(I) \subset H_{\alpha, \beta} \subset E(I)$ where the topology of $H_{\alpha, \beta}$ is stronger than the topology it inherits from $E(I)$ and likewise the topology of $D(I)$ is stronger than the topology it 
inherits from $H_{\alpha, B^{*}}$ Consequently, the inclusion $D^{\star}(I) \supset H_{\alpha, \beta}^{\star} \supset E^{\star}(I)$ holds.

Moreover, if $f(x)$ is a locally integrable function on I such that

$\frac{f(x)}{(1-x)^{-\alpha}(1+x)^{c}} \varepsilon L^{1}(-1,1)$ for any. $1>c>\max (-\beta, 0)$, then $f(x) \varepsilon H_{\alpha, \beta}^{\star}$ and

$$
\langle f, \phi\rangle=\int_{-1}^{1} f(x) \phi(x) d x
$$

for any.$\phi \varepsilon H_{\alpha, \beta}$ since by (3.1) for $k=0$, we have

$$
\begin{aligned}
|\langle f, \phi\rangle| & \leq \int_{-1}^{1}\left|\frac{f(x)}{(1-x)^{-\alpha}(1+x)^{c}}\right|\left|(1-x)^{-\alpha}(1+x)^{c} \phi(x)\right| d x \\
& \leq A \int_{-1}^{1}\left|\frac{f(x)}{(1-x)^{-\alpha}(1+x)^{c}}\right| d x<\infty
\end{aligned}
$$

for some positive constant A.

Evidently,$L$ is a continuous linear operator from $H_{\alpha, \beta}$ into itself since

$$
\gamma_{k, c}(L \phi)=\sup _{x \in I}\left|(1-x)^{-\alpha}(1+x)^{c} L^{k}(L \phi)\right|=\gamma_{k+1, c}(\phi) .
$$

Thus, it is easy to show that the operator $L^{\star}$ defined on $H_{\alpha, \beta}^{\star}$ by

$$
\left\langle L^{*} f, \phi\right\rangle=\langle f, L \phi\rangle ; \quad f \varepsilon H_{\alpha, \beta}^{\star}, \phi \varepsilon H_{\alpha, \beta}
$$

is a continuous linear operator mapping $H_{\alpha, \beta}^{\star}$ into itself.

Moreover, for any fixed $f \in H_{\alpha, \beta}^{\star}$, there exists a positive constant $C$ and a nonnegative integer $p$ such that for any $\phi \in H_{\alpha, \beta}$

$$
|\langle f, \phi\rangle| \leq C \max _{0 \leq k \leq p} \gamma_{k, c}(\phi) \text {. }
$$

Since $p_{\lambda}^{(\alpha, \beta)}(x)=(1-x)^{\alpha}(1+x)^{\beta} p_{\lambda}^{(\alpha, \beta)}(x)$ is a member of $H_{\alpha, \beta}$ as can be seen from (3.1), (2.13) and (2.14), we can define the continuous Jacobi transform $F^{(\alpha, \beta)}(\lambda)$ of any member $f(x)$ of the space $H_{\alpha, \beta}^{\star}$ by

$$
F^{(\alpha, \beta)}(\lambda)=\frac{1}{2^{\alpha+\beta+1}}\left\langle f(x), P_{\lambda}^{(\alpha, \beta)}(x)\right\rangle
$$


Formula (3.6) reduces to (2.19) if $f(x)$ is a locally integrable function on I such that $\frac{f(x)(1-x)^{\alpha}}{(1+x)^{c}} \varepsilon L^{1}(-1,1)$ for any $c$ with $\max (-\beta, 0)<c<1$. Some properties of the continuous Jacobi transform will be given in the following proposition. But first we need the following lemma:

\section{$\underline{\text { Lemma } 3.1}$}

$$
\frac{\mathrm{d}}{\mathrm{d} \lambda}\left(P_{\lambda}^{(\alpha, \beta)}(x)\right) \varepsilon H_{\alpha, \beta} ; \operatorname{Re} \lambda>0
$$

Proof: Since $P_{\lambda}{ }^{(\alpha, \beta)}(x)$ is analytic in $\lambda$ in the region $\operatorname{Re} \lambda>0$ it suffices to show that

$$
\gamma_{k, c}\left(\frac{d}{d \lambda} p_{\lambda}^{(\alpha, \beta)}(x)\right)<\infty
$$

for $k=0,1,2, \ldots$.

We show this by using induction on $k$. First we show that

$$
\gamma_{0, c}\left(\frac{d}{d \lambda} p_{\lambda}^{(\alpha, \beta)}(x)\right)<\infty
$$

i) if $-1<\beta<0$, we write

$$
P_{\lambda}^{(\alpha, \beta)}(x)=W(x) G(\lambda) F\left(-\lambda, \lambda+\alpha+\beta+1 ; \alpha+1, \frac{1-x}{2}\right)
$$

where $W(x)=(1-x)^{\alpha}(1+x)^{\beta}, \quad G(\lambda)=\frac{\Gamma(\lambda+\alpha+1)}{\Gamma(\alpha+1) \Gamma(\lambda+1)}$,

$$
F\left(-\lambda, \lambda+\alpha+\beta+1 ; \alpha+1, \frac{1-x}{2}\right)=\sum_{n=0}^{\infty} a_{n}(\lambda, \alpha, \beta)\left(\frac{1-x}{2}\right)^{n}
$$

and

$$
a_{n}(\lambda, \alpha, \beta)=\frac{\Gamma(-\lambda+n) \Gamma(\lambda+\alpha+\beta+1+n) \Gamma(\alpha+1)}{\Gamma(-\lambda) \Gamma(\lambda+\alpha+\beta+1) \Gamma(\alpha+1+n) \Gamma(n+1)} .
$$

Hence

$$
\frac{d P_{\lambda}^{(\alpha, \beta)}}{d \lambda}=W(x)\left\{G^{\prime}(\dot{\lambda}) F\left(-\lambda, \lambda+\alpha+\beta+1 ; \alpha+1, \frac{l-x}{2}\right)+G(\lambda) \sum_{n=0}^{\infty} \frac{d}{d \lambda} a_{n}(\lambda, \alpha, \beta)\left(\frac{l-x}{2}\right)^{n}\right\} .
$$


Since $F\left(-\lambda, \lambda+\alpha+\beta+1 ; \alpha+1, \frac{1-x}{2}\right)$ is defined and bounded on $[-1,1]$, then

$$
\gamma_{0, c}\left(W(x) F\left(-\lambda, \lambda+\alpha+\beta+1 ; \alpha+1 ; \frac{1-x}{2}\right)\right)<\infty .
$$

By taking the logarithmic derivative of (3.9) we obtain

$$
\begin{aligned}
& \sum_{n=0}^{\infty} \frac{d}{d \lambda} a_{n}(\lambda, \alpha, \beta)\left(\frac{1-x}{2}\right)^{n} \\
= & \sum_{n=0}^{\infty}\left\{\frac{\Gamma^{\prime}(\lambda+\alpha+\beta+1+n)}{\Gamma(\lambda+\alpha+\beta+1+n)}-\frac{\Gamma^{\prime}(-\lambda+n)}{\Gamma(-\lambda+n)}+\frac{\Gamma^{\prime}(-\lambda)}{\Gamma(-\lambda)}-\frac{\Gamma^{\prime}(\lambda+\alpha+\beta+1)}{\Gamma(\lambda+\alpha+\beta+1)}\right\} a_{n}(\lambda, \alpha, \beta)\left(\frac{1-x}{2}\right)^{n} \\
= & I_{1}+I_{2}+\left\{\frac{\Gamma^{\prime}(-\lambda)}{\Gamma(-\lambda)}-\frac{\Gamma^{\prime}(\lambda+\alpha+\beta+1)}{\Gamma(\lambda+\alpha+\beta+1)}\right\} F\left(-\lambda, \lambda+\alpha+\beta+1 ; \alpha+1 ; \frac{1-x}{2}\right) .
\end{aligned}
$$

Since $\frac{\Gamma^{\prime}(\lambda+\alpha+\beta+1+n)}{\Gamma(\lambda+\alpha+\beta+1+n)}=O(\ln (\lambda+\alpha+\beta+1+n))$ and $a_{n}(\lambda, \alpha, \beta)=0\left(\frac{1}{n}\right)$ as $n \rightarrow \infty$, the series defining $I_{1}$ is uniformly and absolutely convergent on $[-1,1]$ and hence

$$
\gamma_{0, c}\left(W(x) I_{1}\right)<\infty .
$$

$I_{2}$ is treated similarly. The last term on the right-hand side of (3.12) has

already been shown to satisfy (3.11). Thus by combining this, $(3.10)$ and $(3.11)$ we obtain that

$$
\gamma_{0, c}\left(\frac{d P_{\lambda}^{(\alpha, \beta)}}{d \lambda}\right)<\infty .
$$

ii) if $\beta=0$, one can show, by using the same argument as in ( $i$ ), that both $F\left(-\lambda, \lambda+\alpha+1 ; \alpha+1 ; \frac{1-x}{2}\right)$ and $\frac{d}{d \lambda} F\left(-\lambda, \lambda+\alpha+1 ; \alpha+1 ; \frac{1-x}{2}\right)$ are $0\left(\ln \frac{1}{1+x}\right)$ as $x \rightarrow-1$, hence they satisfy (3.11) and consequently (3.8) holds.

iii) if $O<B$, we use the following contiguous relation for the hypergeometric function

$$
F(a, b ; c ; z)=(1-z)^{c-a-b} F(c-a, b-a ; c ; z)
$$

to write

$$
p_{\lambda}^{(\alpha, \beta)}(x)=W(x) G(\lambda)\left(\frac{1+x}{2}\right)^{-\beta} F\left(\alpha+\lambda+1,-\lambda-\beta ; \alpha+1 ; \frac{1-x}{2}\right) .
$$


The proof is exactly the same as in part (i) since in the series expansion

$$
\begin{gathered}
F\left(\alpha+\lambda+1,-\lambda-\beta ; \alpha+1 ; \frac{1-x}{2}\right)=\sum_{n=0}^{\infty} a_{n}(\lambda, \alpha, \beta)\left(\frac{1-x}{2}\right)^{n} \\
a_{n}(\lambda, \alpha, \beta)=0\left(\frac{1}{n}\right) \text { as } n \rightarrow \infty .
\end{gathered}
$$

Finally, from (2.6) we have

$$
L_{\lambda}^{k} p_{\lambda}^{(\alpha, \beta)}(x)=[\eta(\lambda)]^{k} p_{\lambda}^{(\alpha, \beta)}(x)
$$

where

$$
n(\lambda)=-\lambda(\lambda+\alpha+\beta+1) .
$$

Therefore,

$$
L^{k} \frac{d}{d \lambda} P_{\lambda}^{(\alpha, \beta)}(x)=p_{\lambda}^{(\alpha, \beta)}(x)\left(\frac{d}{d \lambda}[n(\lambda)]^{k}\right)+[n(\lambda)]^{k} \frac{d}{d \lambda} p_{\lambda}^{(\alpha, \beta)}(x)
$$

and hence

$$
\gamma_{k, c}\left(\frac{d}{d \lambda} p_{\lambda}^{(\alpha, \beta)}(x)\right)=\frac{d}{d \lambda}[n(\lambda)]^{k} \gamma_{0, c}\left(p_{\lambda}^{(\alpha, \beta)}(x)\right)+[n(\lambda)]^{k} \gamma_{0, c}\left(\frac{d}{d \lambda} p_{\lambda}^{(\alpha, \beta)}(x)\right)<\infty .
$$

The last inequality follows from (3.8) and the fact that $P_{\lambda}^{(\alpha, \beta)}(x) \varepsilon H_{\alpha, \beta}$.

$$
\text { Q.E.D. }
$$

Proposition 3.1: Let $f(x) \in H_{\alpha, \beta}^{\star}, F^{(\alpha, \beta)}(\lambda)$ be its continuous Jacobi transform and $\phi(x) \in D(I)$. Then

i) $F^{(\alpha, \beta)}(\lambda)=0\left(\lambda^{q}\right)$ for some $q \geq 0$ as $\lambda \rightarrow \infty$.

ii) $F^{(\alpha, \beta)}(\lambda)$ is analytic in $\operatorname{Re} \lambda>0$ and $\frac{d F^{(\alpha, \beta)}(\lambda)}{d \lambda}=\frac{1}{2^{\alpha+\beta+1}}\left\langle f(x), \frac{d}{d \lambda} p_{\lambda}^{(\alpha, \beta)}(x)>\right.$.

iii) the continuous Jacobi transform $\Phi^{(\alpha, \beta)}(\lambda)$ of $\phi(x)$ given by

$$
\Phi^{(\alpha, \beta)}(\lambda)=\frac{1}{2^{\alpha+\beta+1}} \int_{-1}^{1} \phi(x)(1-x)^{\alpha}(1+x)^{\beta} p_{\lambda}^{(\alpha, \beta)}(x) d x
$$

is rapidly decreasing as $\lambda \rightarrow \infty$, i.e., $\Phi^{(\alpha, \beta)}(\lambda)=0\left(\lambda^{-p}\right)$ for any $p>0$ as 
Proof: i) In view of (3.5), there exists a constant $C$ and a nonnegative integer p such that

$$
\left|F^{(\alpha, \beta)}(\lambda)\right| \leq \frac{1}{2^{\alpha+\beta+1}} c \max _{0 \leq k \leq p} \sup _{x \in I}\left|(1-x)^{-\alpha}(1+x)^{c} L^{k} P_{\lambda}^{(\alpha, \beta)}(x)\right| .
$$

By combining (3.15) and (2.16) we obtain

$$
\begin{aligned}
\left|F^{(\alpha, \beta)}(\lambda)\right| & \leq \frac{1}{2^{\alpha+\beta+1}} C[\ln (\lambda) \mid]^{p} \sup _{x \in I}\left|(1-x)^{-\alpha}(1+x)^{c} p_{\lambda}^{(\alpha, \beta)}(x)\right| \\
& \leq B[\ln (\lambda) \mid]^{p}{ }_{k}(\lambda)
\end{aligned}
$$

where $B$ is a positive constant independent of $\lambda$. But since $n(\lambda)=0\left(\lambda^{2}\right)$ and $k(\lambda)=O\left(\lambda^{q^{\prime}}\right)$ as $\lambda \rightarrow \infty$ where $q^{\prime}=\max (\alpha, \beta)$, it follows that $(i)$ holds for $q=2 p+q^{\prime}$.

ii) With some easy computations one can show that

$$
\begin{gathered}
\frac{F^{(\alpha, \beta)}(\lambda+\Delta \lambda)-F^{(\alpha, \beta)}(\lambda)}{\Delta \lambda}-\frac{1}{2^{\alpha+\beta+1}}\left\langle f(x), \frac{d}{d \lambda} p_{\lambda}^{(\alpha, \beta)}(x)\right\rangle \\
=\frac{1}{2^{\alpha+\beta+1}}\langle f(x), \psi \Delta \lambda(x, \lambda)\rangle
\end{gathered}
$$

where

$$
\psi_{\Delta \lambda}(x, \lambda)=\frac{p_{\lambda+\Delta \lambda}^{(\alpha, \beta)}(x)-p_{\lambda}^{(\alpha, \beta)}(x)}{\Delta \lambda}-\frac{d}{d \lambda} p_{\lambda}^{(\alpha, \beta)}(x) .
$$

By lemma 3.1, $\frac{d}{d \lambda} P_{\lambda}^{(\alpha, \beta)}(x)$ belongs to $H_{\alpha, \beta}$, hence (3.16) makes sense.

The proof will be completed if we can show that

$$
\lim _{\Delta \lambda \rightarrow 0} \psi_{\Delta \lambda}(x, \lambda)=0
$$

in $H_{\alpha, \beta}$. Aga in with some easy computations one can show that

$$
\begin{aligned}
& (1-x)^{-\alpha}(1+x)^{c} L^{k} \Psi_{\Delta \lambda}(x, \lambda) \\
& =(1-x)^{-\alpha}(1+x)^{c}\left\{\frac{[n(\lambda+\Delta \lambda)]^{k} P_{\lambda+\Delta \lambda}^{(\alpha, \beta)}(x)-[n(\lambda)]^{k} p_{\lambda}^{(\alpha, \beta)}(x)}{\Delta \lambda}-L^{k} \frac{d}{d \lambda} P_{\lambda}^{(\alpha, \beta)}(x)\right\}
\end{aligned}
$$


$=(1-x)^{-\alpha}(1+x)^{c}\left\{\frac{[n(\lambda+\Delta \lambda)]^{k} p_{\lambda+\Delta \lambda}^{(\alpha, \beta)}(x)-[n(\lambda)]^{k} p_{\lambda}^{(\alpha, \beta)}(x)}{\Delta \lambda}-\frac{d}{d \lambda}[n(\lambda)]^{k} p_{\lambda}^{(\alpha, \beta)}(x)\right\}$.

But since $[\eta(\lambda)]^{k} p_{\lambda}^{(a, B)}(x)$ is analytic in $\lambda$ it can be expressed as a Cauchy integral and thus we can write (3.18) in the form

$$
\begin{aligned}
& (1-x)^{-\alpha}(1+x)^{c} L^{k} \psi_{\Delta \lambda}(x, \lambda) \\
& =\frac{(1-x)^{-\alpha}(1+x)^{c}}{2 \pi i} \int_{\Gamma} \frac{[n(s)]^{k} p_{s}^{(\alpha, \beta)}(x)}{\Delta \lambda}\left\{\frac{1}{s-(\lambda+\Delta \lambda)}-\frac{1}{s-\lambda}-\frac{\Delta \lambda}{(s-\lambda)^{2}}\right\} d s \\
& =\frac{(1-x)^{-\alpha}(1+x)^{c}}{2 \pi i} \int_{\Gamma} \frac{[n(s)]^{k} p_{s}^{(\alpha, \beta)}(x)}{\Delta \lambda} \frac{(\Delta \lambda)^{2}}{[s-(\lambda+\Delta \lambda)](s-\lambda)^{2}} d s \\
& =\frac{(1-x)^{-\alpha}(1+x)^{c}(\Delta \lambda)}{2 \pi i} \int_{\Gamma} \frac{[n(s)]^{k} p_{s}^{(\alpha, \beta)}(x)}{[s-(\lambda+\Delta \lambda)](s-\lambda)^{2}} d s
\end{aligned}
$$

where $\Gamma$ is a closed contour encircling the points $\lambda, \lambda+\Delta \lambda$ and lying entirely inside the region $\operatorname{Re} \lambda>0$. Let $c_{k}^{\alpha, \beta}=\sup _{\substack{x \in I \\ s \in \Gamma}} \frac{1}{2 \pi}\left|(1-x)^{-\alpha}(1+x)^{c}[n(s)]^{k} p_{s}^{(\alpha, \beta)}(x)\right|$ then, from (3.19) we obtain

$$
\left|(1-x)^{-\alpha}(1+x)^{c} L^{k} \Psi_{\Delta \lambda}(x, \lambda)\right| \leq c_{k}^{\alpha, \beta} A(\Delta \lambda)
$$

where $A=\frac{\ell}{d^{3}}, \quad \ell$ is the length of $\Gamma$ and $d=\min \left(d_{1}, d_{2}\right), d_{1}=\operatorname{distance}(\lambda, \Gamma)$ and $d_{2}=$ distance $(\lambda+\Delta \lambda, \Gamma)$. But the right-hand side of (3.20) approaches zero as $\Delta \lambda \rightarrow \gamma$ and this proves (3.17).

iii) From (3.13) we have

$$
\begin{aligned}
\Phi^{(\alpha, \beta)}(\lambda) & =\frac{1}{2^{\alpha+\beta+1}} \int_{-1}^{1} \phi(x) p_{\lambda}^{(\alpha, \beta)}(x) d x \\
& =\frac{1}{2^{\alpha+\beta+1}} \frac{1}{[n(\lambda)]^{k}} \int_{-1}^{1} \phi(x)[n(\lambda)]^{k} p_{\lambda}^{(\alpha, \beta)}(x) d x \\
& =\frac{1}{2^{\alpha+\beta+1}} \frac{1}{[n(\lambda)]^{k}} \int_{-1}^{1} p_{\lambda}^{(\alpha, \beta)}(x)\left(L^{*}\right)^{k} \phi(x) d x
\end{aligned}
$$

for any nonnegative integer $k$. Thus, 


$$
\left|\Phi^{(\alpha, \beta)}(\lambda)\right| \leq \frac{c}{\left.\ln (\lambda)\right|^{k}}
$$

for some suitable constant $c$ by (2.15). But since $n(\lambda)=O\left(\lambda^{2}\right)$ as $\lambda \rightarrow \infty$, it follows that $\Phi^{(\alpha, \beta)}(\lambda)=O\left(\lambda^{-2 k}\right)$ as $\lambda \rightarrow \infty$ for any nonnegative integer $k$.

Lemma 3.2: Let $f(x) \varepsilon H_{\alpha, \beta}^{*}, F^{(\alpha, \beta)}(\lambda)$ be its continuous Jacobi transform and $\phi(x) \in D(I)$. Let

$$
\tilde{\Phi}^{(\beta, \alpha)}(\lambda)=\int_{-1}^{1} \phi(x) p_{\lambda}^{(\beta, \alpha)}(-x) d x .
$$

Then

i) $\tilde{\Phi}^{(\beta, \alpha)}(\lambda)$ is rapidly decreasing as $\lambda \rightarrow \infty$.

ii) for any fixed real number $r$ with $0<r<\infty$

$$
\begin{aligned}
& \int_{0}^{r} \tilde{\Phi}(\beta, \alpha)\left(\lambda-\frac{1}{2}\right)\left\langle f(y), P_{\lambda-\frac{1}{2}}^{(\alpha, \beta)}(y)\right\rangle H_{0}(\lambda) \lambda \sin \pi \lambda d \lambda \\
& =\left\langle f(y), \int_{0}^{r} p_{\lambda-\frac{1}{2}}^{(\alpha, \beta)}(y) \tilde{\Phi}^{(\beta, \alpha)}\left(\lambda-\frac{1}{2}\right) H_{0}(\lambda) \lambda \sin \pi \lambda d \lambda\right\rangle
\end{aligned}
$$

where $H_{0}(\lambda)$ is given by (2.21).

Proof: i) From (2.3) it is obvious that $P_{\lambda}^{(\beta, \alpha)}(-x)$ satisfies the differential equation

$$
\left(1-x^{2}\right) y^{\prime \prime}-[(\alpha-\beta)+(\alpha+\beta+2) x] y^{\prime}=-\lambda(\lambda+\alpha+\beta+1) y
$$

which by (2.7) can be written as

$$
L^{*} P_{\lambda}^{(\beta, \alpha)}(-x)=-\lambda(\lambda+\alpha+\beta+1) P_{\lambda}^{(\beta, \alpha)}(-x)=\eta(\lambda) P_{\lambda}^{(\beta, \alpha)}(-x) .
$$




$$
\begin{aligned}
\tilde{\Phi}^{(\beta, \alpha)}(\lambda) & =\frac{1}{[n(\lambda)]^{k}} \int_{-1}^{1} \phi(x)[n(\lambda)]^{k} p_{\lambda}^{(\beta, \alpha)}(-x) d x \\
& =\frac{1}{[n(\lambda)]^{k}} \int_{-1}^{1} \phi(x) L^{\star k} p_{\lambda}^{(\beta, \alpha)}(-x) d x=\frac{1}{[n(\lambda)]^{k}} \int_{-1}^{1} p_{\lambda}^{(\beta, \alpha)}(-x) L^{k} \phi(x) d x
\end{aligned}
$$

and from (2.15) we conclude that $\left.\tilde{\Phi}^{(\beta, \alpha)}(\lambda)=0\left(\frac{1}{\lambda}\right)^{2 k}\right)$ as $\lambda \rightarrow \infty$ for any $k \geq 0$.

ii) Let

$$
J_{r}(y)=\int_{0}^{r} p_{\lambda-\frac{1}{2}}^{(\alpha, \beta)}(y) \tilde{\Phi}^{(\beta, \alpha)}\left(\lambda-\frac{1}{2}\right) H_{0}(\lambda) \lambda \sin \pi \lambda d \lambda .
$$

Evidently, $J_{r}(y) \in H_{\alpha, \beta}$, for

$$
\begin{gathered}
\gamma_{k, c}\left(J_{r}(y)\right)=\sup _{y \in I}\left|(1-y)^{-\alpha}(1+y)^{c} \int_{0}^{r}\left[\eta\left(\lambda-\frac{1}{2}\right)\right]^{k} p_{\lambda-\frac{1}{2}}^{(\alpha, \beta)}(y) \tilde{\Phi}^{(\beta, \alpha)}\left(\lambda-\frac{1}{2}\right) H_{0}(\lambda) \lambda \sin \pi \lambda d \lambda\right| \\
\leq \int_{0}^{r} \gamma_{0, c}\left(p_{\lambda-\frac{1}{2}}^{(\alpha, \beta)}(y)\right)\left|\left[n\left(\lambda-\frac{1}{2}\right)\right]^{k}\right|\left|\tilde{\Phi}^{(\beta, \alpha)}\left(\lambda-\frac{1}{2}\right) H_{0}(\lambda) \lambda\right| d \lambda<\infty .
\end{gathered}
$$

In fact, $J_{\infty}(y)$ is also in $H_{\alpha, \beta}$ as can be seen from (3.25) and the

first part of this lemma since $H_{0}(\lambda)=0\left(\frac{1}{\lambda^{\alpha+\beta}}\right)$ as $\lambda \rightarrow \infty$. Construct the following Reimann sum for the integral $J_{r}(y)$

$$
R_{m}\left[J_{r}(y)\right]=\frac{r}{m} \sum_{k=1}^{m} P_{\frac{k r}{m}-\frac{1}{2}}^{(\alpha, \beta)}(y) \tilde{\Phi}^{(\beta, \alpha)}\left(\frac{k r}{m}-\frac{1}{2}\right) H_{0}\left(\frac{k r}{m}\right)\left(\frac{k r}{m}\right) \sin \left(\frac{\pi k r}{m}\right) .
$$

As a function of $y$, each term in this sum belongs to $H_{\alpha, \beta}$, hence

$\left\langle f(y), R_{m}\left[J_{r}(y)\right]\right\rangle$ is defined and in addition to that we have

$$
\left\langle f(y), R_{m}\left[J_{r}(y)\right]\right\rangle=\frac{r}{m} \sum_{k=1}^{m}\left\langle f(y), p_{\frac{k r}{m}-\frac{1}{2}}^{(\alpha, \beta)}>h_{k}(r, m)\right.
$$

where

$$
h_{k}(r, m)=\tilde{\Phi}(\beta, \alpha)\left(\frac{k r}{m}-\frac{1}{2}\right) H_{0}\left(\frac{k r}{m}\right)\left(\frac{k r}{m}\right) \sin \left(\frac{\pi k r}{m}\right) .
$$

Upon observing that the sum in (3.26) converges to the left hand side of (3.22) as $m \rightarrow \infty$, our lemma will be proved if we can show that $R_{m}\left[J_{r}(y)\right] \rightarrow J_{r}(y)$ in $H_{\alpha, \beta}$ as $m \rightarrow \infty$. Let 


$$
h(\lambda)=\tilde{\Phi}^{(\beta, \alpha)}\left(\lambda-\frac{1}{2}\right) H_{0}(\lambda) \lambda \sin (\pi \lambda) \text {. }
$$

Then

$$
\begin{aligned}
& \gamma_{p, c}\left(J_{r}(y)-R_{m}\left[J_{r}(y)\right]\right)=\sup _{y \in I} \mid(1-y)^{-\alpha}(1+y)^{c} L^{p}\left\{\int_{0}^{r} P_{\lambda-\frac{1}{2}}^{(\alpha, \beta)}(y) h(\lambda) d \lambda-\frac{r}{m} \sum_{k=1}^{m} p_{\frac{k r}{m}-\frac{3}{2}}^{(\alpha, \beta)}(y) h_{k}(r, m)\right\} \\
& =\sup _{y \in I}\left|(1-y)^{-\alpha}(1+y)^{c}\left\{\int_{0}^{r}\left[n\left(\lambda-\frac{1}{2}\right)\right]^{p} p_{\lambda-\frac{1}{2}}^{(\alpha, \beta)}(y) h(\lambda) d \lambda-\frac{r}{m} \sum_{k=1}^{m}\left[\frac{k r}{m}-\frac{1}{2}\right]^{p} p_{\frac{k r}{m}-\frac{1}{2}}^{(\alpha, \beta)}(y) h_{k}(r, m)\right\}\right| \\
& =\sup _{y \in I} \mid(1-y)^{-\alpha}(1+y)^{c}\left\{\sum_{k=1}^{m} \int_{\frac{(k-1) r}{m}}^{m}\left[n\left(\lambda-\frac{3}{2}\right)\right]^{p} p_{\lambda-\frac{3}{2}}^{(\alpha, \beta)}(y) h(\lambda) d \lambda-\frac{r}{m} \sum_{k=1}^{m}\left[\frac{k r}{m}-\frac{3}{2}\right]^{p} p_{\frac{k r}{m}-\frac{3}{2}}^{(\alpha, \beta)}(y) h_{k}(r, m)\right\} \\
& =\sup _{y \in I}\left|(1-y)^{-\alpha}(1+y) c\left\{\sum_{k=1}^{m}\left[\int_{\frac{(k-1) r}{m}}^{\frac{k r}{m}}\left[n\left(\lambda-\frac{1}{2}\right)\right]^{p_{p}}{ }_{\lambda-\frac{1}{2}}^{(\alpha, \beta)}(y) h(\lambda) d \lambda-\frac{r}{m}\left[\frac{k r}{m}-\frac{1}{2}\right]^{p} p_{\frac{k r}{m}-\frac{1}{2}}^{(\alpha, \beta)}(y) h_{k}(r, m)\right]\right\}\right|
\end{aligned}
$$

But since $(1-y)^{-\alpha}(1+y)^{c}\left[n\left(\lambda-\frac{1}{2}\right)\right]^{p} P_{\lambda-\frac{3}{2}}^{(\alpha, \beta)}(y) h(\lambda)$ is a uniformly continuous function of $(y, \lambda)$ in the region $y \in[-1,1]$ and $\lambda \in[0, r]$, it follows that for any $\varepsilon>0$, there exists $m_{0}$ large enough so that

$$
\gamma_{p, c}\left(J_{r}(y)-R_{m}\left[J_{r}(y)\right]\right) \leq \sum_{k=1}^{m} \varepsilon\left(\frac{r}{m}\right) \leq \varepsilon r
$$

for any $m>m_{0}$ and this proves our assertion.

4. Characterization of the continuous Jacobi transform:

Q.E.D.

The main result of this section is theorem 4.1 which gives a characterization of the continuous Jacobi transforms of members of the space $H_{\alpha, \beta}^{\star} ; \alpha+\beta=0$, $-1<\alpha \beta<1$.

Theorem 4.1. A necessary and sufficient condition for a function $F(\lambda)$ to be the continuous Jacobi transform of some generalized function in the space $H_{\alpha, \beta}^{\star}$ according to definition (3.6) is that $F(\lambda)$ is analytic in the half-plane $\operatorname{Re} \lambda>0$ and bounded according to 


$$
|F(\lambda)| \leq Q(|\lambda|), \quad \operatorname{Re} \lambda>0
$$

where $Q(|\lambda|)$ is a polynomial in $|\lambda|$.

Proof: Necessity: Was proved in proposition 3.1. Sufficiency: Let $q=\operatorname{deg} Q$ and choose two positive integers $m$ and $n$ such that $2 m-n>q$ with $n$ greater than the $\max \left\{\frac{3}{2}, 2-\min (\alpha, \beta)\right\}$.

Since

$$
\left|\sqrt{\lambda}\left[n\left(\lambda-\frac{1}{2}\right)+1\right]^{-m} F\left(\lambda-\frac{1}{2}\right)\right| \leq c \lambda^{-2 m+q+\frac{1}{2}}=C \lambda^{\frac{1}{2}-n}
$$

for some positive constant $C$ and sufficiently large $\lambda$, it follows that

$$
\int_{b}^{\infty} \frac{\sqrt{\lambda} F\left(\lambda-\frac{1}{2}\right)}{\left[n\left(\lambda-\frac{1}{2}\right)+1\right]^{m}} d \lambda<\infty \text { for any } b>0,
$$

and since the integrand is continuous and bounded on $[0, b], \frac{\sqrt{\lambda} F\left(\lambda-\frac{3}{2}\right)}{\left[n\left(\lambda-\frac{3}{2}\right)+1\right]^{m}} \varepsilon L^{l}(0, \infty)$. Therefore,by (2.19) and (2.20)

$$
\frac{F\left(\lambda-\frac{1}{2}\right)}{\left[n\left(\lambda-\frac{3}{2}\right)+1\right]^{m}}=\frac{3}{2} \int_{-1}^{1} g(x) p_{\lambda-\frac{1}{2}}^{(\alpha, \beta)}(x) d x
$$

where

$$
g(x)=4 \int_{0}^{\infty}\left[n\left(\lambda-\frac{3}{2}\right)+1\right]^{-m} F\left(\lambda-\frac{3}{2}\right) P_{\lambda-\frac{3}{2}}^{(\beta, \alpha)}(-x) H_{0}(\lambda) \lambda \sin \pi \lambda d \lambda .
$$

Hence,

$$
\sup _{x \in I}\left|(1-x)^{\alpha} g(x)\right| \leq 4 \int_{0}^{\infty}\left|\left[n\left(\lambda-\frac{1}{2}\right)+1\right]^{-m} F\left(\lambda-\frac{1}{2}\right) \kappa(\lambda) H_{0}(\lambda) \lambda\right| d \lambda
$$

where

$$
\kappa(\lambda)=\sup _{x \in I}\left|(1-x)^{\alpha} P_{\lambda-\frac{1}{2}}^{(\beta, \alpha)}(-x)\right|
$$

But since

$$
\left|\left[n\left(\lambda-\frac{1}{2}\right)+1\right]^{-m} F\left(\lambda-\frac{1}{2}\right) \kappa(\lambda) H_{0}(\lambda) \lambda\right| \leq C \lambda^{-2 m+q} \lambda^{\max (\alpha, \beta)} \lambda^{-\alpha-\beta+1} \leq C \lambda^{-n-m i n(\alpha, \beta)+1}
$$

for some suitable constant $C$, it follows from our choice of $n$ that the integral 
in $(4.1)$ is finite and hence $\sup _{x \in I}\left|(1-x)^{\alpha} g(x)\right|<\infty$. Therefore, $\frac{(1-x)^{\alpha} g(x)}{(1+x)^{c}} \varepsilon L^{1}(-1,1)$ for any $0<c<1$ which implies that $g(x) \varepsilon H_{\alpha, \beta}^{*}$ by $(3.2)$.

Now set

$$
f(x)=\left(L^{*}+1\right)^{m} g(x)
$$

which is also in $H_{\alpha, \beta}^{\star}$ since $L^{*}$ is a continuous linear operator on $H_{\alpha, \beta}^{\star}$; see (3.3) and (3.4).

finàlly

$$
\begin{gathered}
\frac{3}{2}\left\langle f(x), P_{\lambda-\frac{3}{2}}^{(\alpha, \beta)}(x)\right\rangle=\frac{1}{2}\left\langle\left(L^{\star}+1\right)^{m} g(x), P_{\lambda-\frac{1}{2}}^{(\alpha, \beta)}(x)\right\rangle \\
=\frac{3}{2}\left\langle g(x),(L+1)^{m} P_{\lambda-\frac{1}{2}}^{(\alpha, \beta)}(x)\right\rangle=\frac{1}{2}\left[n\left(\lambda-\frac{1}{2}\right)+1\right]^{m}\left\langle g(x), P_{\lambda-\frac{1}{2}}^{(\alpha, \beta)}(x)\right\rangle \\
=\left[n\left(\lambda-\frac{1}{2}\right)+1\right]^{m}\left[n\left(\lambda-\frac{1}{2}\right)+1\right]^{-m} F\left(\lambda-\frac{1}{2}\right)=F\left(\lambda-\frac{1}{2}\right) .
\end{gathered}
$$

Q.E.D.

\section{Inversion of the Jacobi transform:}

The bulk of this section is devoted to deriving an inversion formula for the Jacobi transforms of generalized functions. This inversion formula, of course, generalizes the classical one given in [ 5$]$.

Since the inversion formula for the continuous Jacobi transform given by (2.19) and (2.20) are only valid for $\alpha+\beta=0$, from now on we shall always assume that this is indeed the case.

Lemma 5.1. Let $\phi(y) \varepsilon D(I)$ and $\tilde{\Phi}^{(\beta, \alpha)}(\lambda)$ be given as in (3.21). Then

$$
2 \int_{0}^{r} P_{\lambda-\frac{1}{2}}^{(\alpha, \beta)}(y) \tilde{\phi}^{(\beta, \alpha)}\left(\lambda-\frac{1}{2}\right) H_{C}(\lambda) \lambda \sin \pi \lambda d \lambda+\phi(y)
$$


Fronf: Lei

$$
\phi_{r}(y)=2 \int_{0}^{r} P_{\lambda-\frac{1}{2}}^{(\alpha, \beta)}(y) \tilde{\Phi}^{(\beta, \alpha)}\left(\lambda-\frac{1}{2}\right) H_{0}(\lambda) \lambda \sin \pi \lambda d \lambda
$$

Clearly, $\lim _{r \rightarrow \infty} \phi_{r}(y)$ exists in view of lemma 3.2 part (i).

Let

$$
\psi(x)=\frac{\phi(x)}{(1-x)^{\alpha}(1+x)^{\beta}}
$$

and $\hat{\psi}^{(\alpha, \beta)}(n)$ be its $n$th Jacobi coefficient as given by $(2.17)$. Hence,

$$
\psi(x)=\sum_{n=0}^{\infty} \frac{1}{h_{n}} \hat{\psi}^{(\alpha, \beta)}(n) P_{n}^{(\alpha, \beta)}(x)
$$

where the series converges absolutely and uniformly an I since $\psi \varepsilon D(I)$. By combining (3.21) and (5.3), we obtain

$$
\begin{aligned}
\Phi^{(\beta, \alpha)}\left(\lambda-\frac{1}{2}\right) & =\int_{-1}^{1}(1-x)^{\alpha}(1+x)^{\beta} \psi(x) p_{\lambda-\frac{1}{2}}^{(\beta, \alpha)}(-x) d x \\
& =\sum_{n=0}^{\infty} \frac{1}{h_{n}} \hat{\psi}^{(\alpha, \beta)}(n) \int_{-1}^{1}(1-x)^{\alpha}(1+x)^{\beta} p_{n}(\alpha, \beta)(x) p_{\lambda-\frac{1}{2}}(\beta, \alpha)(-x) d x
\end{aligned}
$$

which, in virtue of $(2.8)$ and $(2.11)$, yields

$$
{ }_{\Phi}(\beta, \alpha){ }_{\left(\lambda-\frac{1}{2}\right)}=2 \sum_{n=0}^{\infty} \frac{(-1)^{n}}{h_{n}} \dot{\psi}^{(\alpha, \beta)}(n) \hat{p}_{\lambda-\frac{1}{2}}^{(\beta, \alpha)}(n)
$$

where the series converges absolutely and uniformly in $\lambda$ on any compact subset of $[0, \infty)$ since $\left\{\hat{\psi}^{(\alpha, \beta)}(n)\right\}_{n=0}^{\infty}$ is a rapidly decreasing sequence. By substituting (5.4) into (5.2) and interchanging the integration and the summation, one obtains

$$
\phi_{r}(y)=4 \sum_{n=0}^{\infty} \frac{(-1)^{n}}{h_{n}} \hat{\psi}^{(\alpha, \beta)}(n) \int_{0}^{r} P_{\lambda-\frac{1}{2}}^{(\alpha, \beta)}(y) \hat{p}_{\lambda-\frac{1}{2}}^{(\beta, \alpha)}(n) H_{0}(\lambda) \lambda \sin \pi \lambda d \lambda
$$

i) If $-1<\beta<\frac{1}{2}\left(-\frac{1}{2}<\alpha<1\right)$, we use (2.12) to obtain

$$
p_{\lambda-1,2}^{(\alpha, t)}(y)=\sum_{n=0}^{a} \prod_{n} \hat{p}_{\lambda-\frac{1}{2}}^{(a, F)}(n) p_{n}^{(\alpha, \beta)}(y)
$$

where $h_{n}$ and $\hat{p}_{\lambda-1}^{(a, j)}(n)$ are given by (2.10) and (2.11) respectively. The series in (5.6) converges absolutely and uniformly on any compact subset of I because of (2.15) and the fact that $h_{n}=0\left(\frac{1}{n}\right)$ and $\hat{p}_{\lambda-\frac{1}{2}}^{(\alpha, \beta)}(n)=0\left(\frac{1}{n^{2-\beta}}\right)$ as $n \rightarrow \infty$.

By substituting (5.6) into (5.5) and interchanging the integration and the 
summation, we obtian

$$
\phi_{r}(y)=4 \sum_{n, m=0}^{\infty} \frac{(-1)^{n}}{h_{n} h_{m}} \hat{\psi}^{(\alpha, \beta)}(n) P_{m}(\alpha, \beta)(y) I_{r}(m, n)
$$

where $\quad I_{r}(m, n)=\int_{0}^{r} \hat{P}_{\lambda-\frac{1}{2}}^{(\alpha, \beta)}(m) \hat{P}_{\lambda-\frac{1}{2}}^{(\beta, \alpha)}(n) H_{0}(\lambda) \lambda \sin \pi \lambda d \lambda$

But Since

$$
\lim _{r \rightarrow \infty} I_{r}(m, n)=\frac{(-1)^{n}}{4} n_{n} \delta_{n, m} \quad(c . f .[3])
$$

we deduce from (5.7) and (5.3) that

$$
\begin{aligned}
\lim _{r \rightarrow \infty} \phi_{r}(y) & =\sum_{n=0}^{\infty} \frac{1}{h} \hat{\psi}_{n}^{(\alpha, \beta)}(n) P_{n}(\alpha, \beta)(y)=(1-y)^{\alpha}(1+y)^{\beta} \sum_{n=0}^{\infty} \bar{h}_{n} \hat{\psi}^{(\alpha, \beta)}(n) P_{n}^{(\alpha, \beta)}(y) \\
& =\phi(y)
\end{aligned}
$$

ii) If $-1<\alpha<\frac{1}{2}\left(-\frac{1}{2}<\beta<1\right)$, we can, with some easy calculations, use (2.19) and (2.20) with $f(x)=p_{n}^{(\alpha, \beta)}(x)$ to reduce (5.5) to (5.8)

Interchanging the sumation and the limit is possible since the series converge uniformly on any compact subset of $(-1,1)$.

To show that $\phi_{r}(x) \rightarrow \phi(x)$ in $H_{\alpha, \beta}$ as $r \rightarrow \infty$ we need to show that $\gamma_{k, c}\left(\phi_{r}-\phi\right) \rightarrow 0$ as $r \rightarrow \infty$ for every nonnegative integer $k$ and $1>c>\max (-\beta, 0)$.

First, taking a note of (3.23) we make the following observation.

$$
\begin{aligned}
& \left(L_{y} p_{\lambda}^{(\alpha, \beta)}(y)\right) p_{\lambda}^{(\beta, \alpha)}(-x)=n(\lambda) p_{\lambda}^{(\alpha, \beta)}(y) p_{\lambda}^{(\beta, \alpha)}(-x) \\
= & p_{\lambda}^{(\alpha, \beta)}(y)\left(n(\lambda) p_{\lambda}^{(\beta, \alpha)}(-x)\right)=p_{\lambda}^{(\alpha, \beta)}(y)\left(L^{\star} p_{\lambda}^{(\beta, \alpha)}(-x)\right) .
\end{aligned}
$$

From this, it easily follows that

$$
L_{y} K_{r}(y, x)=L_{x}^{\star} K_{r}(y, x)
$$

Therefore, for any positive integer $k$ we have 


$$
\begin{aligned}
L_{y}^{k} \phi_{r}(y) & =2 \int_{-1}^{1} \phi(x) L_{y}^{k} K_{r}(y, x) d x=2 \int_{-1}^{1} \phi(x) L_{x}^{\star k} K_{r}(y, x) d x \\
& =2 \int_{-1}^{1}\left(L_{x}^{k} \phi(x)\right) K_{r}(y, x) d x .
\end{aligned}
$$

But since $L^{k} \in \mathcal{D}(I)$, we can apply (5.1) to it and conclude that

and consequently

$$
\lim _{r \rightarrow \infty} L_{y}^{k} \phi_{r}(y)=L_{y}^{k} \phi(y)
$$

$$
\gamma_{k}\left(\phi_{r}-\phi\right)+0 \text { as } r \rightarrow \infty \text {. }
$$

Q.E.D.

Now'we are able to prove our main result.

Theorem 5.1. Let $f(x) \in H_{\alpha, \beta}^{\star}$ and $F^{(\alpha, \beta)}(\lambda)$ be its continuous Jacobi transform. Then in the sense of convergence in $D^{\star}(I)$

$$
f(x)=\lim _{n \rightarrow \infty} 4 \int_{0}^{n} F(\alpha, \beta)\left(\lambda-\frac{1}{2}\right) P \underset{\lambda-\frac{1}{2}}{(\beta, \alpha)}(-x) H_{0}(\lambda) \lambda \sin \pi \lambda d \lambda .
$$

Proof: Let $\phi(x) \in D(I)$ and

$$
f_{n}(x)=4 \int_{0}^{n} F(\alpha, \beta)\left(\lambda-\frac{1}{2}\right) \underset{\lambda-\frac{1}{2}}{(\beta, \alpha)}(-x) H_{0}(\lambda) \lambda \sin \pi \lambda d \lambda .
$$

We need to show that $\left\langle f_{n}, \phi\right\rangle+\langle f, \phi\rangle$ as $n+\infty$.

By changing the order of integration and using lemma 3.2 we obtain

$$
\begin{aligned}
\left\langle f_{n}, \phi\right\rangle & =4 \int_{0}^{n} F(\alpha, \beta)\left(\lambda-\frac{1}{2}\right) \tilde{\phi}^{(\beta, \alpha)}\left(\lambda-\frac{1}{2}\right) H_{0}(\lambda) \lambda \sin \pi \lambda d \lambda \\
& =2 \int_{0}^{n}\left\langle f(u), \underset{\lambda-\frac{1}{2}}{p(\alpha, \beta)}(u)\right\rangle \tilde{\Phi}^{(\beta, \alpha)}\left(\lambda-\frac{1}{2}\right) H_{0}(\lambda) \lambda \sin \pi \lambda d \lambda \\
& =\left\langle f(u), 2 \int_{0}^{n}{ }^{p}(\alpha, \beta)(u) \tilde{\phi}(\beta, \alpha)\left(\lambda-\frac{1}{2}\right) H_{0}(\lambda) \lambda \sin \pi \lambda d \lambda\right\rangle
\end{aligned}
$$


where $\tilde{i}(\tilde{f},(1)$ is given by (3.21). Interchanging the order of the integration is permitted since $\phi(x)$ has a compact support in $I$ and the integrand is a continuous function on a closed bounded domain. Taking the limit of both sides of $(5.10)$ as $n \rightarrow \infty$ yields

$$
\lim _{n \rightarrow \infty}\left\langle f_{n}, \phi\right\rangle=\langle f, \phi\rangle
$$

by lemma 5.1 .

Q.E.D.

An immediate consequence of theorem 5.1 is the following weak version of a uniqueness theorem:

Corollary 5.1. Let $f(x), g(x) \in H_{\alpha, \beta}^{\star}$ and let $F^{(\alpha, \beta)}(\lambda)$ and $G^{(\alpha, \beta)}(\lambda)$ be their continuous Jacobi transforms respectively. If $F^{(\alpha, \beta)}(\lambda)=G^{(\alpha, \beta)}(\lambda)$ in $\operatorname{Re} \lambda>0$, then in the sense of equality in $D^{\star}(I), f=g$. Corollary 5.2. Let $f(x)$ be a regular member of $H_{\alpha, \beta}^{\star}$ such that $f(x) \varepsilon \cdot H_{\alpha, \beta}^{\star} \cap L \cdot(-1,1)$, then

$$
f(x)=4 \int_{0}^{\infty} F(\alpha, \beta)\left(\lambda-\frac{1}{2}\right) P_{\lambda-\frac{1}{2}}^{(\beta, \alpha)}(-x) H_{0}(\lambda) \lambda \sin \pi \lambda d \lambda
$$

for almost all $x \in(-1,1)$.

Acknowledgement: The author wishes to thank professor L. Debnath for bringing to his attention the results of references [1] and [3]. 


\section{REFERENCES}

1. Butzer, P., Stens, R. and Wehrens, M. The Continuous Legendre Transform, its Inverse Transform and Applications, Internat. J. Math. and Math. Sci. 3(1980), 47-67.

2. Camphe11, L. A Comparison of the Sampling Theorems of Kramer and Whittaker, Soc. of Indust. Appl. Math. 12 (1964), 117-130.

3. Debnath, L. Jacobi Transform, Bul1. Cal. Math. Soc. 55 (1963), 113-121.

4. Debnath, L. Solution of Partial Differential Equations by the Method of Jacobi Transform, Bul1. Cal. Math. Soc. 59 (1967), 155-158.

5. Deeba, E. and Koh, E. The Continuous Jacobi Transform, Internat. J. of Math. and Math. Sci. 6 (1983), 145-160.

6. Erdelyi, A., Magnus, W., Oberhettinger, F. and Tricomi, F. Higher Transcendental Functions, Vol. 1, McGraw Hill Book Company, New York, 1953.

7. Jerri, A. On the Application of Some Interpolating Functions in Physics, J. Res. Nat. Bur. Stands, Ser. B, Math. Sci., 73 B (1969), 241-245.

8. Jerri, A. Sampling Expansion for the $L_{v}^{\alpha}$-Laguerre Intearal Transform, J. Res. Nat. Bur. Stands, Ser. B, Math. Sci., V $80 B(1976), 415-418$.

9. Kramer, H. A Generalized Samplina Theorem, J. Math. Phy. 38 (1959), 68-72.

10. Zemanian, A. Genralized Integral Transformations, John Wiley and Sons, New York (1968). 


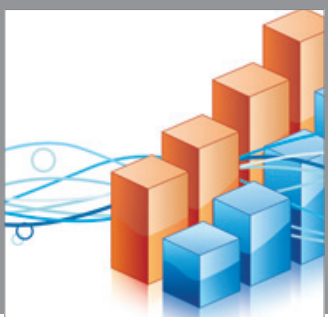

Advances in

Operations Research

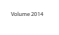

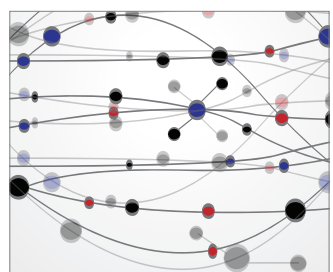

\section{The Scientific} World Journal
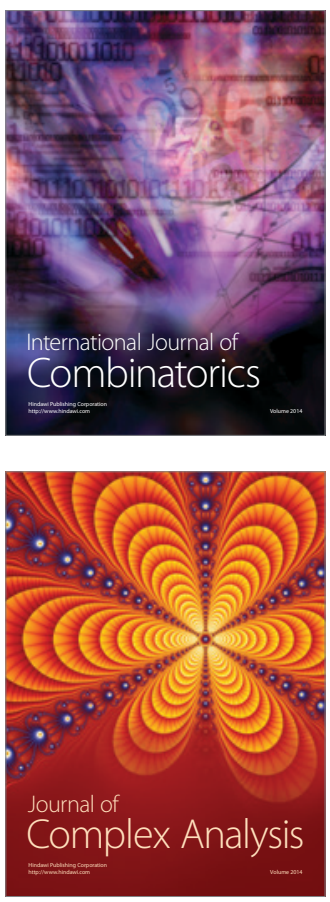

International Journal of

Mathematics and

Mathematical

Sciences
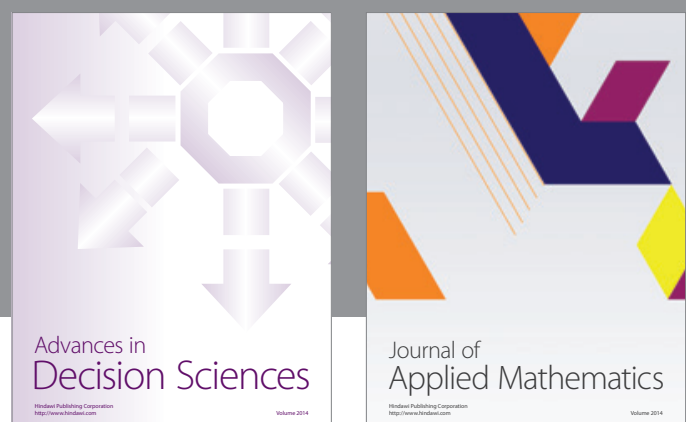

Journal of

Applied Mathematics
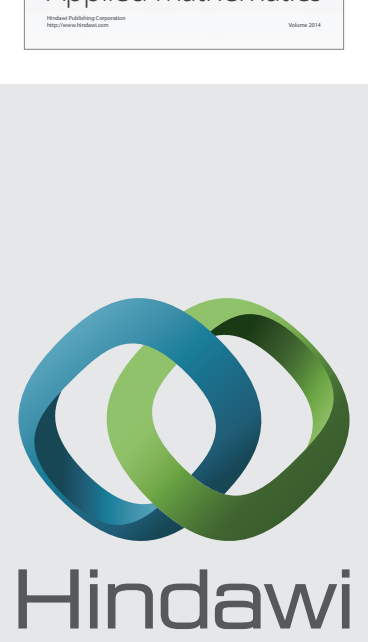

Submit your manuscripts at http://www.hindawi.com
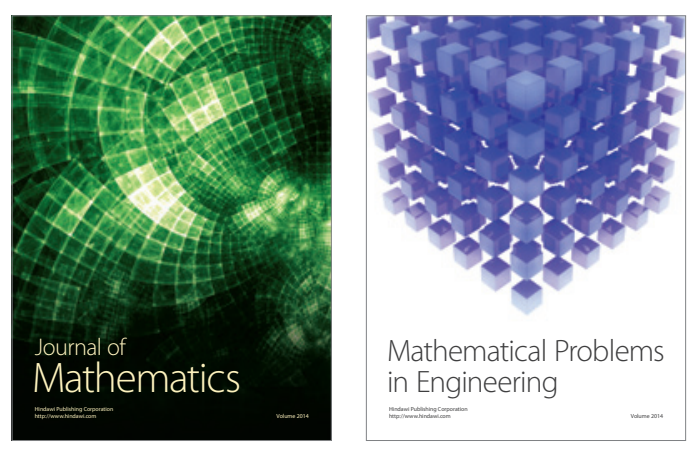

Mathematical Problems in Engineering
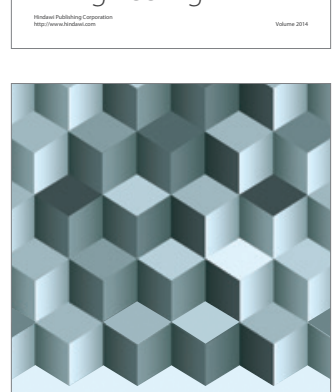

Journal of

Function Spaces
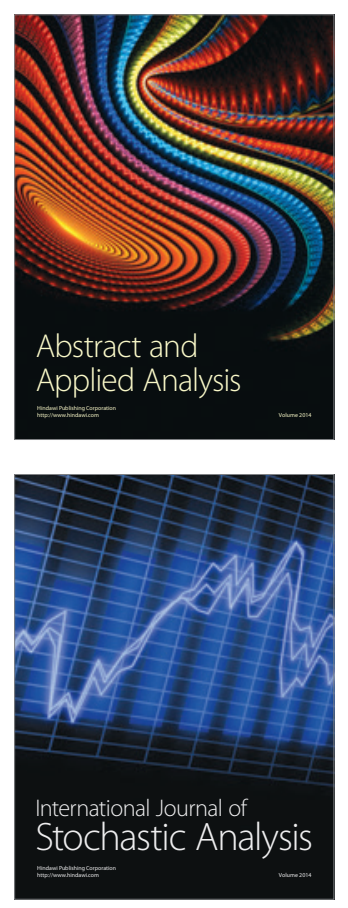

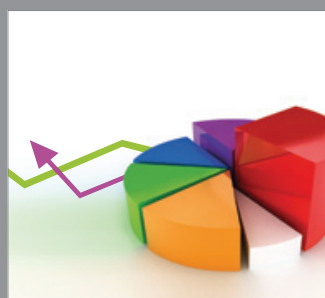

ournal of

Probability and Statistics

Promensencen
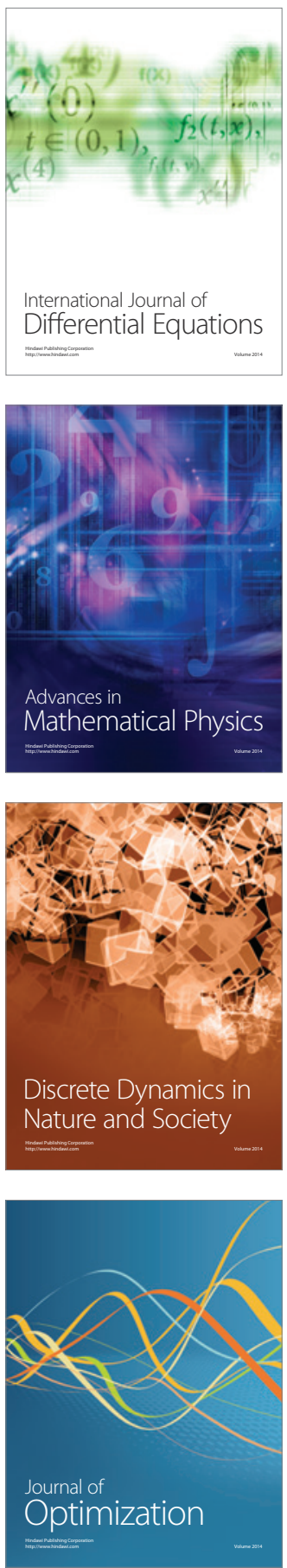